\title{
The use of energy saving technology as the basis for the state program implementation
}

\author{
Nadezhda Novopashina ${ }^{1, *}$, and Svetlana Puring ${ }^{1}$ \\ ${ }^{1}$ Samara State Technical University, Institute of Architecture and Civil Engineering, 194 \\ Molodogvardeyskaya St., 443001 Samara, Russia
}

\begin{abstract}
The article views prospects of application of energy-saving technology in heat sources and plants that consume thermal energy in the form of steam and hot water. This technology involves creation of a twophase medium in the transonic jet nozzle apparatus. The effectiveness of the transonic jet nozzle apparatus is compared with the recuperative heat exchangers effectiveness. The use of these devices allows to obtain economic benefit by reducing the required building volume to accommodate the transonic jet nozzle apparatus, increasing performance coefficient compared with conventional recuperative heat exchangers and reducing operating costs. Two operation modes are considered: "pumpboiler" and "boiler". The most effective is "pump-boiler" mode, because, in this case, more energy saving is obtained through acting powerful network pumps only as launchers. An example of the use of transonic jet nozzle devices in the Samara region is given in the article. The trends and opportunities to solve the task controlling thermobaric parameters by changing the volume fraction of steam in the two-phase medium are studied.
\end{abstract}

\section{Introduction}

Given the sharp increase in energy prices, structural and technological redevelopment of production activities on the basis of new energy-saving technologies, allowing to abandon the metal-intensive, inefficient, outdated equipment should become the main priority of reforms in energy-consuming industries [1-2].

The Russian government in its Resolution (further GR) № 321 of April 15, 2014 approved the State program of the Russian Federation titled Energy Efficiency and Energy Development including the Energy Conservation and Energy Efficiency sub-program. The aim of this sub-program is the implementation of energy-saving technology on sources of heat and enterprises consuming thermal energy in the form of steam and hot water, and optimal use of energy resources [3-6] in the production activities of industrial enterprises.

In general, as the practice of the thermal energy evaluation produced by boilers, and power plants shows, it does not pay income received from consumers taking into account

*Corresponding author: novopashina2010@yandex.ru 
the subsidies. Studies have shown the need for evaluation of external market factors: pricing, consumer solvency and other. There is a need in a more flexible approach to the customer and the development of payment options for obtained thermal energy.

It is obvious that there is a foremost need, above all, to bring small heat sources out of the state control and transfer them for rent or other type of property to private entrepreneurs who will implement modernization elements into the process of operation of thermal energy sources and reduce the number of managing and maintenance staff to a minimum. This will reduce the cost of produced and supplied heat energy.

One of the weakest spots in the heat source is the reliability of the steam heaters for hot water heating systems. Lack of funds for water treatment and poor operating characteristics leads to frequent failure of the pipe system of heaters and it also leads to failure of industrial and housing heating. On the inner surface of the pipe system there appears lime scale, and open area for passage of secondary heat transfer fluid (hot water) is reduced. The coefficient of efficiency, which is $98 \%$ for the new heat exchangers, is greatly reduced during the operation, which increases the cost of supplied thermal power. The existing steam and water-cooled recuperative heat exchangers with the length of 6 meters or more require large space and building area on heat sources not only for their own accommodation, but also for the repair and total renovation. All this hinders the implementation of the Government Resolution.

One of the promising directions for the implementation of the GR is the use of transonic jet nozzle apparatus (further TJA) [7], where two-phase steam-water mixture with certain thermobaric parameters is created by mixing steam and water (primary and secondary coolant).

\section{Materials and Methods}

In terms of heat engineering, they are contact (mixing) heat exchangers, in which water (or other liquid) is heated with saturated steam. They are most effective in terms of energy and resource saving compared to the recuperative heat exchangers applied at heat sources. Standard size apparatuses have been developed with the diameter from 25 to $100 \mathrm{~mm}$, thermal capacity up to $14 \mathrm{Gcal} / \mathrm{h}$, the length of which does not usually exceed $70 \mathrm{~cm}$. The small size allows embedding them in virtually any existing industrial thermal scheme or heat source using or generating saturated steam [7]. The main amount of lime scale is formed in the accelerating tube with the length up to $2.5 \mathrm{~m}$, which is located immediately after the devices. Maximum formation of lime scale on the walls occurs at a water temperature of about $60^{\circ} \mathrm{C}$. In the case of deposition of a large amount of lime scale all the current or total renovation includes replacing the accelerating line.

TJA can operate in two modes:

- "Boiler" mode means that the apparatus only heats the water and its pumping is performed by network pumps. In this mode, the pumping effect of apparatuses is minimal. However, there is a need to maintain sufficient water pressure before the network pumps in order to avoid cavitation in the pump inlet nozzle and to ensure that the water temperature does not exceed acceptable limit for the pump;

- "Pump - boiler" mode: the device is constructed and designed so that the secondary fluid is heated and there is a surge (sudden increase) of pressure of steam-water mixture, by means of which its transportation is carried out. To start the apparatuses, it is necessary to provide flow of secondary coolant through them with existing network pumps, and then to pass steam. After achieving the calculated mode (temperature rise) and the emergence of a surge (increase in pressure in the accelerating line), network pumps must be switched off, i.e., network pumps operate only during the start-up. This results in energy savings.

As an example, let us consider the work of TJA in the heating system with a 
temperature schedule of $150 / 70{ }^{\circ} \mathrm{C}$ with a constant flow of heated water (line 1) and quality control for the residential areas of the Samara region, with an estimated heating outside air temperature $-30^{\circ} \mathrm{C}$ (Figure 1). Changing water temperature at the inlet to the apparatus $T_{1}$ (line 3) and at the exit from the apparatus after heating $T_{2}$ (line 2) makes a linear dependence with the outdoor temperature $T_{2}$. The heating season begins in autumn, when the outdoor temperature falls below $+8^{\circ} \mathrm{C}$ and stays so for at least five days. Under such circumstances, according to the temperature schedule, the flow temperature is about $45^{\circ} \mathrm{C}$ in the inlet pipe, and in the return pipe it is about $33^{\circ} \mathrm{C}$, i.e. temperature difference is $12^{\circ} \mathrm{C}$. With the calculated heating temperature $\left(-30^{\circ} \mathrm{C}\right)$ the difference will be $80^{\circ} \mathrm{C}$. Thus, the difference in water temperature at the outlet of the device and at the inlet to it varies from 12 to $80^{\circ} \mathrm{C}$ (line 4) throughout the heating season.

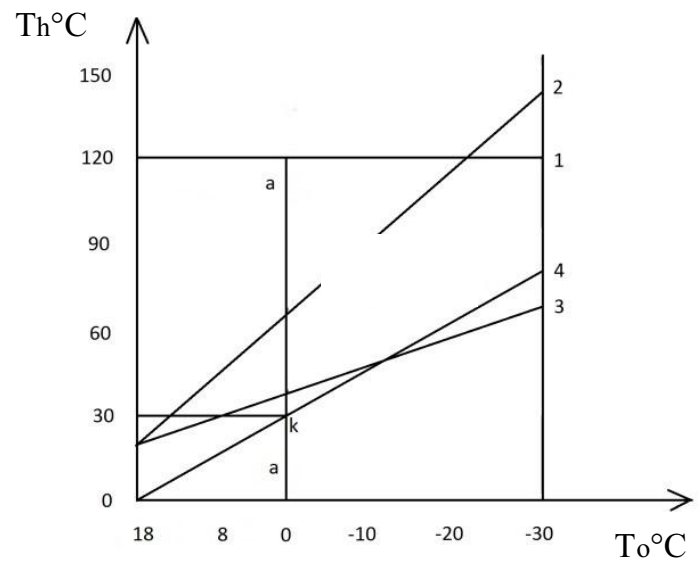

Fig. 1. Temperature Schedule of Apparatuses Operation. 1. Water flow $V, \mathrm{~m}^{3} / \mathrm{h}$. 2. Water temperature at the inlet $T_{1},{ }^{\circ} \mathrm{C}$. 3 . Water temperature at the outlet $T_{2},{ }^{\circ} \mathrm{C} .4$. The difference in water temperature in the apparatus $T_{1}-T_{2},{ }^{\circ} \mathrm{C}$.

\section{Results}

As shown by full-scale study [7, 8], for the stable operation of the apparatuses, calculated individually for each object, it is necessary to maintain three main requirements in the "pump-boiler" mode:

- Heating of the secondary fluid by at least $30^{\circ} \mathrm{C}$;

- The pressure of saturated water steam should be in the range from 30 to $100 \%$ of the calculated. When steam pressure is less than $30 \%$ of the calculated, the apparatus can only work in the "boiler" mode;

- The flow rate must be greater than the speed of sound to obtain a pressure surge.

In accordance with the above, on the graph 1 let us draw from the point " $\mathrm{k}$ " a vertical line a-a, corresponding to the temperature difference between the supply and return coolant temperature of $30^{\circ} \mathrm{C}$. This line divides the chart into two areas: to the right of this line there is an area of apparatuses operation in the "pump - boiler" mode, to the left - in the "boiler" mode.

Similar temperature schedules for TJA apparatuses operation can be made for other temperature graphs.

Figure 2 shows the dependence of the sound speed in the homogeneous two-phase mixture from the volume phase ratio, which can be represented as 


$$
\beta=\frac{v_{n}}{\left(v_{n}+v_{\varkappa}\right)}
$$

where $v_{n}$ is the amount of steam in the mixture; $\left(v_{n}+v_{\varkappa}\right)$ is the mixture volume.

The speed of sound in the water reaches $1500 \mathrm{~m} / \mathrm{sec}$, in clean steam it makes from 300 to $480 \mathrm{~m} / \mathrm{sec}$, depending on the steam parameters.

As seen from the graph, the sound speed decreases sharply in the range of ratios of steam-water $0.1-0.2$, and likewise increases in the range of ratios steam-water $0.8-1$. If water and steam are mixed in a ratio of $02-08$, creating homogenous two-phase mixture, the speed of sound in it on some sources is sharply reduced to $5-20 \mathrm{~m} / \mathrm{sec}$. Pressure surge occurs when the mixture flow rate passes the sound barrier, which is easy enough to set up in practice.

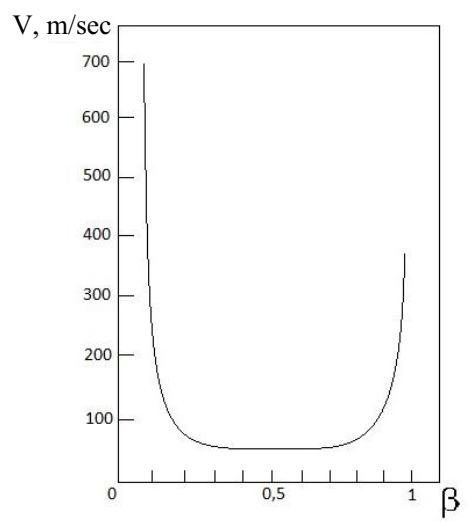

Fig. 2. The Dependence of the Speed of Sound in the Two-Phase Mixture from Volume Phase Ratio.

The basis of the apparatus operation is the fact that the local speed of sound in homogeneous two-phase mixture of water and steam is substantially less than sound speed in each of the separate phases. As a result, in the water-steam mixture the effect of supersonic flow is easily achieved [8-11] and at the outlet abrupt changes in temperature and pressure parameters occur, allowing TJA to work in "pump-boiler" mode. There is theoretical and practical interest in determining the dependence of these parameters on the speed of sound in a homogeneous two-phase medium and the ability to manage them by changing the speed of sound, or changing the ratio of water-steam.

According to [12], the speed of sound in homogeneous two-phase steam-water flow is determined by the formula

$$
a=\frac{a_{n}}{\sqrt{\left.\left[(1-\beta)\left(v_{n} / v_{b}\right)+\beta\right)\right] \cdot\left[(1-\beta)\left(k_{n} / k_{b}\right)+\beta\right]}},
$$

where $a_{n}$ is the speed of sound in the dry saturated steam; $k_{n}, k_{b}$ are adiabatic performance of steam and water at saturation line; $v_{n}, . v_{b}$ are values of specific volumes of water and steam at saturation line; $\beta$ - steam content in the two-phase medium (volume ratio of steam and water).

In the case of an incompressible fluid, when $k_{n} / k_{b}=0$, this formula is written as

$$
a=\frac{a_{n}}{\sqrt{\left.\left[(1-\beta)\left(v_{n} / v_{b}\right)+\beta\right)\right] \cdot \beta}} .
$$


Considering that the value $v_{b}$ is almost constant, at the minimum value of $\beta=0,5$ the speed of sound will depend on the specific volume of steam $v_{n}$, i.e. on steam pressure.

As shown in [12], the value $\beta$ corresponding to the minimum speed of sound in the two-phase mixture of an incompressible fluid looks like

$$
\beta_{\text {min }}=0.5\left[1+\frac{1}{v_{n} / v_{b}-1}\right] \approx 0.5 \text {. }
$$

This equation goes quite well with Figure 2.

Let us substitute the value $\beta_{\min }$ into the formula (2). Let us set $\frac{v_{n}}{v_{b}}=x$. Then

$$
a_{\min }=\frac{a_{n}}{\sqrt{\left[\left(1-0.5\left[1+\frac{1}{x-1}\right]\right) \cdot x+0.5\left[1+\frac{1}{x-1}\right]\right] \cdot 0.5\left[1+\frac{1}{x-1}\right]}} .
$$

After transformation we get

$$
a_{\min }=\frac{2 a_{n} \cdot \sqrt{v_{n} / v_{b}-1}}{v_{n} / v_{b}}
$$

\section{Discussion}

Equation (3) to determine the value $\beta$ counts only the specific volumes of water and steam at saturation line, and corresponds to the minimum value of speed of sound in a two-phase mixture of an incompressible fluid; it apparently assumes that water and steam are mixed in equal amounts.

Given that the speed of sound in homogeneous two-phase steam-water flow depends on the values relation of specific volumes of water and steam at saturation line, i.e. of state parameters, it is possible to obtain different values of the sound speed by creating various parameters of water and steam state prior to mixing.

The calculations following these formulas showed that the minimum values of the sound speed for these mixtures depend on the steam pressure and range from 32 to $54 \mathrm{~m} /$ $\mathrm{sec}$, which is much more than the values given above $(5-20 \mathrm{~m} / \mathrm{sec})$.

After mixing water with steam in the apparatus there appears homogeneous two-phase mixture, the speed of which reaches at the annulus inlet $50 \mathrm{~m} / \mathrm{sec}$ [13-16]. Mach number $\mathrm{M}=\frac{\omega}{\alpha}$, which is a flow rate ratio of the speed of sound in this stream, will not exceed 1.5. Given that the intensity of the pressure surge (from $\mathrm{P}_{1}$ to $\mathrm{P}_{2}$ ) is proportionate to the square of the Mach number, the pressure after the surge in the apparatus may be several times higher than the pressure of steam and water before it, i.e. the ratio $\mathrm{P}_{2} / \mathrm{P}_{1}$ can reach 2.25 and the apparatus can operate in the "boiler-pump" mode. If the steam pressure before the apparatus is $0.4 \mathrm{MPa}$, then we can achieve output pressure of $0.9 \mathrm{MPa}$. 
In the apparatus Mach number $M>1$ is achieved by reducing the speed of sound at relatively low flow rates, thus reducing energy consumption for creation of the pressure surge and the coolant pumping.

In addition, the use of apparatuses TJA on heat sources and small enterprises using saturated steam to create an individual heat source will yield cost savings by:

-Reducing capital expenditures, as the required volume of construction will significantly decrease and available volume can be used for other purposes;

-Reducing operational costs, as TJA routine maintenance requires considerably less time than standard routine maintenance of recuperative heat exchangers;

- Reducing energy consumption during apparatuses operation in the "pump - boiler" mode.

\section{Conclusions}

Thus, one of the most promising directions for the implementation of the Russian Federation Government Resolution is the use of energy-saving technology and transonic jet nozzle apparatus TJA for heating systems, which will reduce energy consumption and improve energy efficiency of heat sources.

\section{References}

1. G.I. Titov, N.A. Novopashina, V.G. Titov, Urban Construction and Architecture 2, 1922 (2016). doi: 10.17673/Vestnik.2016.02.4

2. D.N. Vatuzov, S.M. Puring, E.B. Filatova, N.P. Tyurin, Urban Construction and Architecture 4, 86-91 (2014). doi: 10.17673/Vestnik.2014.04.13

3. M. E. Deich, V. S. Danilin, V. N. Shanin, G. V. Tsiklauri, Thermal Engineering 6, 7679 (1969)

4. M.N. Galimzyanov, S.A. Lepikhin, Bulletin of the Samara State University 2 (76), 96104 (2010)

5. V. V. Fissenko, Critical Two-Phase Flows (Moscow, 1978)

6. D. Chisholm, Two-Phase Flow in Pipelines and Heat Exchangers, 204 (Moscow, 1986)

7. N.A. Novopashina, I.A. Blatov, Scientific Review 4,146-149 (2014)

8. N.A. Novopashina, G.I. Titov, Bulletin of the Samara State Technical University, Series: Engineering 2 (30), 200-205 (2011)

9. V.S. Shagapov, I.K. Gimaltdinov, M.N. Galimzyanov, Fluid Mechanics 2, 139-147 (2002)

10. V.T. Sitenkov, Theory and Calculation of Two-Phase Systems, 204 (Nizhnevartovsk, 2006)

11. E.E. Blagov, Valve Industry 6 (45), 44-49 (2006)

12. E.E. Blagov, Valve Industry 4 (49), 45-52 (2007)

13. E.E. Blagov, Valve Industry 4 (43), 55-59 (2006)

14. E. E. Blagov, Valve Industry 2 (41), 37-42 (2006)

15. E. E. Blagov, Science and Construction 4, 55-59 (2006)

16. V. A. Stenin, E. G. Lebedeva, Academic Journal of Western Siberia, Tyumen 2, 73-74 (2011) 